\title{
USING RECURSIVE DISTANCE VECTOR METHODOLOGY TO REVIEW REMOTE DESKTOP SOLUTIONS IN THE SMALL BUSINESS CONSULTING ENVIRONMENT
}

\author{
Stephen Hinton, East Carolina University, shinton@triad.rr.com
}

\begin{abstract}
The scope of this paper is twofold. They are to survey remote access solutions for the small business support environment, and to examine a methodology for making complex multivariate business decisions. The particular needs of a small business technology consultant are examined to select which solutions would be considered in the review process. Log Me In Pro, TeamViewer, and WebEx Remote Access were chosen as 3 potential solutions that fit the basic needs of the environment based on their ability to create an active listening connection that would work in heterogeneous network environments. The benefits of a good decision making framework are discussed, as well as the value of data visualization. The methodology for using a recursive distance vector approach for comparing potential solutions was explained. For the specific application of the methodology to this problem the top-tier variables selected were cost, utility, and security. The cost variable was subdivided into 3 scenarios to give a broad idea of how costs would scale over time and with varying number of users and computers. The utility variable combined the concepts of end user ease of use in an attended access scenario, and the features of the solution for the remote support consultant. Security was ranked as an aggregate without sub-dividing the variable further. It demonstrated use of the methodology, showing the use of recursion, aggregate tiers, and combining tiers with a variable number of sub-tiers into a decision model. Given this model a stakeholder can plug in their own preferences and rankings about any number of business decisions and get a concise summarized result, as well as being able to visualize the competing benefits and factors along each step of the process.
\end{abstract}

Keywords: remote desktop, remote access, data visualization, recursive distance vector, small business, decision making, Log Me In Pro, TeamViewer, WebEx Remote Access

\section{INTRODUCTION}

Remote desktop access software can be used in a variety of ways in a modern computing environment.. However, their most common use is as a way to reduce per desktop support costs. According to Dassanayaka et al. [6], "Developing a system that helps system administrators to perform their administration task more effectively and efficiently is of great importance to reduce downtime, cost and manpower requirement." This paper is going to evaluate options currently available in the market for a specific niche, the small business support role. Further, this paper will outline the use of a recursive distance vector approach to evaluating options that can be applied to many different business decisions.

Small business support offers a particularly difficult environment for making many IT decisions. When a business is too small to dedicate internal resources to technology support, a common solution is to contract out that work to an outside vendor. A small business support provider must keep overhead costs low to justify their expense. They cannot take advantage of many 'economies of scale' features to dilute cost. Features such as multiple system integration or network resource streamlining are not of benefit [3]. At the same time the external support provider will be providing technology support in any number of dissimilar computing environments. So a solution that will work with multiple operating systems, network, and security configurations is an important consideration. Lastly, the reason for outsourcing the work to begin with is the lack of technical prowess internal to the supported environment. As such each product deployed must have the ease of use for the end user as a significant concern.

The needs of the small business support environment drove the selection of products for review in this paper. From an enterprise perspective the most common remote access solutions are Terminal Services/Remote Desktop protocol from Microsoft, XenDesktop from Citrix, or one of the many implementations of the native Unix protocol VNC [7]. However, in all of these instances the deployment scenario depends on creating a server within the network to maintain connections and licenses. The network firewall must be configured to allow communication to that server 


\section{Issues in Information Systems}

Volume 13, Issue 1, pp. 94-104, 2012

if external access is desirable. This adds a burden to the computing environment in terms of hardware, and complex configuration [19]. It must be maintained for each separate network environment. While the benefits of such a server are evident in large scale deployments; they are cumbersome for a small business.

The primary feature that was important in selecting a product for small business support was an active listening setup. The application installed on the target computer, the one to be controlled, initiates an outbound connection to an external source. The external source then keeps track of the target computers availability, and creates a session to the controlling computer as necessary. Because the initial connection is outbound from the target network perspective it is generally unnecessary to adjust the edge firewall, or configure an onsite server to manage connections. This allows an external consultant to install the software on computers that need to be managed at any number of locations, under differing computing environments, and still connect to a single source on the internet to manage those client connections.

Even with this restriction there are a large number of software solutions that might fit the bill. It would be impractical in the confines of this paper to evaluate all possible solutions. Popularity of the software, reviews from other sources, and real world experience of the author of this paper were used to select three solutions for consideration $[5,13,14]$. This paper will consider LogMeIn Pro, TeamViewer, and WebEx Remote Access.

The technology field is full of multivariable choices similar to the selection of remote desktop software. There are often multiple solutions available, and many seemingly independent decision factors that must be considered. Humphrey [8] makes the argument this way, "One of the primary elements in the construction industry that truly separates the successful companies from the not-so-successful companies is the ability to make good, effective decisions. And yet it's one of those areas of our businesses that we usually don't spend a lot of time thinking about. Oh, we think about the decisions we make, just not about how we make them." As such a methodology for evaluation your options can be extremely valuable. It is very likely that within a few months of this paper's publication there will be new versions of the software evaluated being published. In addition, new vendors may enter the market or rise to prominence. Technology evolves rapidly, so the ability to quickly reassess choices has value.

Furthermore, when there are multiple factors affecting a decision the ability to visualize the competing criteria can greatly enhance the ability of stakeholders to comprehend the problem and come to a good decision. Miller [12] says , "Graphical representation of data is a rapid, concise and effective way of communicating complex information. Or, to rephrase, a picture is worth a thousand words." Often the various criteria for any given choice will be subjective, intangible and hard to quantify. The recursive distance vector approach allows those involved in the decision process to assign a simple and effective quantitative value and integrate it into the evaluation.

\section{METHODOLOGY}

The recursive distance vector method allows a stakeholder to quickly evaluate any number of options along multiple criteria. It assigns a simple quantitative value through the use of ranking. It allows the inclusion of any number of criteria through recursion. The use of distance vectors results in an objective final result that can also be easily visualized with simple graphs.

The initial concept to this methodology is the use of ranking to assign quantitative values. Given $\mathrm{N}$ alternative solutions to a problem you should rank them for each criterion from worst to best. An integer value can be assigned to each rank with the worst option having a value of 0 , and the best option a value of $\mathrm{N}-1$.

This simple system of ranking from worst to best accomplishes two goals. First it puts all quantitative measures on a single comparable scale. Factors such as cost, memory size, installation time can all be given definite objective numeric values on which to evaluate. However, if both cost and memory size are important criterion then it is not trivial compare dollars to kilobytes. But comparing $1^{\text {st }}, 2^{\text {nd }}$ and $3^{\text {rd }}$ is intuitive. Furthermore, many decision criteria will not have an easy to identify numerical value. As Stated by Bryson and Ngwenyama [2], "In practice many 


\section{Issues in Information Systems}

Volume 13, Issue 1, pp. 94-104, 2012

criteria are qualitative and the decision maker can only express ordinal preferences by stating which of a pair of alternatives he prefers most." Factors such as quality, customer service, or warranty may not be easy to quantify with precision. But a ranking scale does not require precision. It only requires a simple preference.

Once all solutions have been ranked according to all criteria it is time to combine and evaluate these factors. By assigning a single non-negative value to each criteria you can consider each solution as a coordinate in a Cartesian space, i.e. $\langle x, y, z\rangle$. Furthermore, with each criteria using the larger values for better alternatives then the coordinate furthest from the origin point $\langle 0,0,0\rangle$ is the 'best' overall option. Therefor the distance formula gives us a simple way to combine all criteria into a single number by which to evaluate each solution [1].

$\mathrm{d}=\overline{\left(x_{2}-x_{1}\right)^{2}+\left(y_{2}-y_{1}\right)^{2}+\cdots+\left(z_{2}-z_{1}\right)^{2}}$

However, the formula can be simplified further since in all instances we are attempting to find the distance from the origin point.

$\mathrm{d}=\overline{x^{2}+y^{2}+\cdots+z^{2}}$

Additionally, up to this point we have assumed that all criteria are equally important. This assumption will likely be valid in most evaluations. Careful selection of criteria generally results in factors of roughly equal consideration. However, in instances where one factor is more important than the others you can add a weight variable to the distance formula to 'skew' the result towards along one axis. Assume variables A, B, and C represent the relative weights of criteria $\mathrm{x}, \mathrm{y}$, and $\mathrm{z}$. Then the coordinates would be of the form $\langle\mathrm{Ax}, \mathrm{By}, \mathrm{Cz}\rangle$ and would alter the distance formula accordingly.

$\mathrm{d}_{\text {weighted }}=\overline{(A x)^{2}+(B y)^{2}+\cdots+(C z)^{2}}$

If the number of criterion can be limited to 3 or less another significant benefit can be realized. Each alternative solution can be mapped onto a three dimensional graph for the stakeholders to visualize and evaluate the alternatives. However, confining any business decision to 3 or less factors would be very limiting.

The solution to wanting more than 3 factors, but not wanting to sacrifice visualization of the data is the use of the recursion concept. Consider all of the factors that would be good to include in the decision, and then try to 'group' the factors so that you can create 'tiers' of roughly equivalent value. For instance you may want to consolidate capital expenditure, training, and recurring costs into a single criterion called expenses. For the lowest tier of each branch you use the ranking system to generate your coordinate values. For each recursive tier use the result of the distance formula to rank the alternatives. In this way you can consider any number of criteria, by nesting them in tiers of 3 criteria or less. At each tier in the hierarchy the data can be expressed visually to aid in understanding the available options. By ranking at each new branch of the hierarchy you preserve the equivalent value of the criteria in lower tiers.

It is important that at each step in the recursive process you once again rank the alternatives based on the result of the distance vector. The distance of an individual vector can exceed its rank. If you simply plugged the result of the distance formula into higher levels they would gain influence based on placement in the hierarchy. Tiers with more sub-branches would have larger vectors. Ranking at each tier eliminates this skew.

\section{DATA \& ANALYSIS}

Based on the needs of small business support we need to choose our top tier variables to consider. The first area to consider will be cost. The economic factor is a variable in almost every business decision. Secondly, the solutions will be evaluated in terms of their utility. This factor will combine the ease of use on the end user and the features available to the support consultant during connection. No matter how you approach remote access you cannot get 


\section{Issues in Information Systems}

Volume 13, Issue 1, pp. 94-104, 2012

around the fact that you are providing a new threat vector to the computer's security. As such, mitigating the risk with good security measures is an important consideration. So the criteria of cost, ease of use, and security will be the top-tier criteria for the recursive distance vector method.

\section{Cost}

While cost is nearly an invariable consideration in any business decision, it is not always straight forward.. The solution providers all have various business models. Some sell licenses by the number of 'servers', 'clients', or users. Some have single-purchase price, others are based on an ongoing subscription cost. We are going to use the recursive nature of the methodology to break down the cost factor in three ways, and use the result of the distance vector formula as to rank our alternatives for final consideration. Considering three scenarios will gives us a feel for how costs scale with both time and the number of hosts supported.

\section{$I^{\text {st }}$ month scenario}

The first cost factor will be first month for one client. We are going rank our solutions based on the initial cost of a single license to connect one support user to one remote computer. This factor will not consider trial period length. One month is the common shortest term for the subscription rates for the products being considered. Log Me In Pro uses a subscription based model per host computer. It costs $\$ 9.99$ per month per computer [4]. TeamViewer uses a lifetime subscription based on the number of hosts installed. A lifetime license can be had for \$749.00[9]. WebEx Remote Access also uses a subscription based model based on the number of hosts. However, their pricing information is not published with their product information. I obtained pricing information in email correspondence with sales representative Michael Haddad in April of 2012. A license to remotely control a single host is $\$ 5 /$ month but with a minimum purchase of 10 licenses. The first month cost for a single host will be $\$ 50.00$ Based on these figures we assign ranks of : Log Me In Pro (2), TeamViewer (1), WebEx Remote Agent (0).

\section{5 year scenario}

The second scenario that we will use as a variable is a 5 users connecting to 5 computers over the course of 5 years. Log Me In Pro does not consider users in its pricing model. However, there is a volume discount at 5 hosts for $\$ 249.00$ per year (\$49.80/host) [4]. The total cost for Log Me In Pro under this scenario is $\$ 1,245.00$. TeamViewer does not have a volume discount, so for 5 hosts the total cost is \$1,444.00[9]. WebEx Remote Access costs extended to 5 years is $\$ 3,000.00$. Rankings under this scenario are: Log Me In Pro (2), TeamViewer (1), WebEx Remote Access (0).

\section{0 year scenario}

The last scenario that we will use as a variable is a 10 users connecting to 10 computers over the course of 10 years. Log Me In Pro introduces another volume discount at 10 licenses (\$44.90/computer) [4]. The total cost for Log Me In Pro under this scenario is $\$ 4,490.00$. TeamViewer Premium allows an unlimited number of agents to control an unlimited number of hosts[9]. At $\$ 1,499.00$ for a lifetime license it is the obvious choice for this scenario. WebEx Remote Access costs extended to 10 years is $\$ 6,000.00$ and under this scenario you are using all of your minimum purchase of 10 hosts. Rankings under this scenario are: Log Me In Pro (1), TeamViewer (2), WebEx Remote Access (0).

\section{Cost Summation}

By considering the costs of each solution under 3 different scenarios we have a better understanding of the real total cost of ownership for each solution. Applying the distance vector formula to each product we can come up with the cost ranking. 


\section{Issues in Information Systems}

Volume 13, Issue 1, pp. 94-104, 2012

$\log$ Me In Pro $=\overline{2^{2}+2^{2}+1^{2}}=3$

TeamViewer $=\overline{1^{2}+1^{2}+2^{2}}=2.4494897427831780981972840747059$

WebEx Remote Access $=\overline{0^{2}+0^{2}+0^{2}}=0$

We can also visually asses the various cost factors in a simple graph.

\section{Utility}

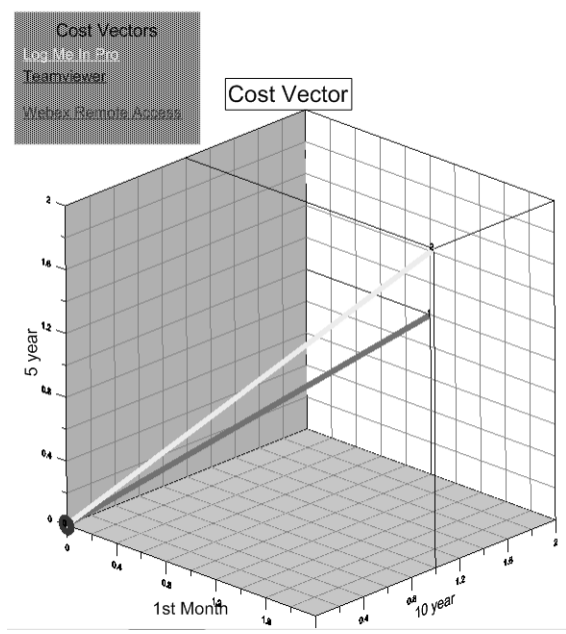

The second variable for evaluation is the utility of each solution. This is an aggregate variable combining two important factors in the consideration into a single top-tier vector. The second tier variables are end user ease of use and support features available during the connection.

\section{Ease of Use}

As noted during the introduction one of the driving factors for small business IT consulting decision is the lack of internal technical expertise in the supported environment. As such it is important that any solution selected for this environment does not place an undue technical burden on the end user. All three solutions can be set up for unattended access. In this configuration the start of a remote control session requires no action or even notification of the end user. However, for reasons of security and computer resources it may not be ideal to leave the software configured for unattended access at all times. Therefore, the difficulty of initiating or allowing a session when the software is configured for attended access is a more important decision factor.

Log Me In Pro is set up for unattended access by default. However, the option to disable or enable access is an easy to use feature. If access is currently disabled, enabling requires two-steps. First the user must start the software. Secondly, the welcome screen displays a clear message about the state of the software with a button labeled 'Turn on now' to allow access. Once this button has been pressed the computer is available for remote control. No further action is necessary by the end user. If $\log \mathrm{Me}$ In Pro is configured to require the host to consent to each remote control session, there is a simple 'allow' prompt at the start of the remote session. The Log Me In account holder can now initiate the connection from the external website. However, the end user can also send an invitation for remote access from within the software. This is also an easy process guided by a wizard that creates an email to with a web link that is sent on the user's behalf. This email is sent independent of the user's own email client or settings and therefore is not dependent on other software being correctly configured. Invitations sent in this manner are also time-sensitive, preventing this from being exploited from a security standpoint [10]. 


\section{Issues in Information Systems}

Volume 13, Issue 1, pp. 94-104, 2012

TeamViewer is installed for attended access by default. As such, remote access is enabled automatically when the software is started. However, unlike the other two solutions there is not a central website for the support consultant to initiate the session. Instead, TeamViewer randomly generates a 9 digit ID code and a password. These must be communicated to the support agent in some manner. The TeamViewer software does not have an integrated invitation or notification method for the user to use. They must communicate these pieces of information independently, via phone, email etc. Once the remote session is initiated no additional consent by the end user is required. They are notified of the remote session, and can disconnect if desired. However, no further active participation is required by the end user after starting the software [15].

WebEx Remote Access is configured for unattended access by default as well. Like TeamViewer, simply starting the software is sufficient to enable remote access control. To disable remote access it is necessary to close the software from the taskbar. The agent can be configured to require approval at the start of each remote session. The prompt to allow control is a simple one. Sessions are initiated by the support consultant from the external website. There is no function built into the software for the host user to initiate or invite a connection [17].

Given these three configurations we can rank the solutions. Assuming the easiest configuration for attended access the easiest software to initiate is WebEx Remote Access, as simply starting the software is the only step for the user. Deciding on the order of the other two components is a little more complex. Team viewer only needs to be started, whereas Log Me In Pro requires two steps to start the software and enabled access. However, Log Me In Pro automatically communicates its available status to an external source. TeamViewer requires that the user communicate its status, ID and password in some manner external to the software. As a result the rankings are: WebEx Remote Support (2), Log Me in Pro (1), TeamViewer (0).

\section{Features}

Getting the remote session started is an important hurdle, but it is only the first step. This brings us to the next variable for consideration, the features available during connection. In many ways this is the real heart of the matter. This is the decision factor that most considers how the solution works. It is also one that demonstrates the utility of the ranking system, as this is a consideration that is difficult to quantify. But all that is required for the decision making methodology is expressing a simple preference through ranking. All three solutions share many features in common, such as supporting chat and file transfers. We are going to examine each solution for some of it's more unique and useful features.

Log Me In Pro has several resources available to the support consultant that are not available in the other solutions. Most prominent is a large array of remote system information. From the dashboard available during the connection you can see the remote system CPU and Memory status, list of drives, scheduled tasks, installed applications, network connections, etc. This is a very extensive collection of data that may be useful to a technician in the diagnoses of problems. There is extensive control over the relative size, resolution, and appearance of the remote host screen. The most unique of which is a magnifier tool that lets the remote use increase the size of one portion of the screen for detail. The ability to synchronize or exchange clipboard data is also very useful for cutting and pasting from one computer to the next. Approximately 40 special keyboard combinations can be transmitted to the host computer. The Log Me In agent can be installed on Windows or Mac based computers. It also has special viewer apps for the Android and iOS mobile operating systems [10].

TeamViewer has a lighter user interface. Yet, it maintains all the most important capabilities and has a few unique benefits. Rather than having a few preset key combinations there is a feature that can be toggled on an off to send all keyboard combinations to the host computer. This is a useful feature as not all special combinations can be anticipated by the designers. All of the solutions have some capacity to restart the host machine, but TeamViewer can request that the host machine restart in safe mode. TeamViewer integrates a VPN driver into it's software installation and thus has the ability to create a virtual tunnel from local to remote machine. This can be an important tool for a support technician attempting to diagnose issues with network resources rather than the individual host. 


\section{Issues in Information Systems}

Volume 13, Issue 1, pp. 94-104, 2012

Lastly, TeamViewer allows the local and remote control computers to 'switch sides'. This interesting feature would be rarely used in a support context, but might be useful in collaborative efforts. TeamViewer can be installed on Windows, Mac, or Linux computers. It has viewer applications for those operating systems and Android and iOS mobile devices[15].

WebEx Remote Access is the lightest of the three solutions on additional features available during connection. It is still a useful tool, but it does not have the capability to take a screenshot of the remote computer, and CTRL-ALTDEL is the only special key combination that it can transmit to the remote computer. It does have an annotation feature that lets the remote user make highlights, text notes, and drawings on the remote screen. This essentially turns the entire desktop into a whiteboard. This could be very useful for training or user education. Additionally, WebEx Remote Access has the ability to invite additional users into a remote desktop session. This feature could be used for collaboration, or when third party support needs to be consulted on an issue. The last unique feature of this solution is the ability to remotely control computers that have not been previously prepared. Using the WebEx Remote Support feature of the website allows the support technician to send an invitation email to any user requesting remote control of their desktop. The receiving user must be able to receive the email, have a working web browser, and an installed Java runtime environment. This list of requirements, and the burden placed on the end user may be problematic. However, the capability to control any computer instead of only specified hosts will be of tremendous value in some environments [17].

The ranking of the features of each solution is inescapably subjective. However, the intent of any decision making model is not to take the decision out of the hands of the stakeholders. Instead, the intent is to provide a framework for making the decision and to simplify the process. As such the ranking below is based on the previous work experience and subjective judgment of the paper's author. For purposes of this paper the preference ranking for software feature are: Log Me In Pro (2), TeamViewer (1), WebEx Remote Access (0).

Utility Summation

Considering the ease of use for the end use and the features valuable to the support consultant give us a good overview of the utility of each solution. The distance vector formula can be used with two variables to create a composite rank for use in the top-tier comparison.

$\log$ Me In Pro $=\overline{1^{2}+2^{2}}=2.2360679774997896964091736687313$

TeamViewer $=\overline{0+1^{2}}=1$

WebEx Remote Access $=\overline{2^{2}+0^{2}}=2$

Again, we can use a simple graph to visualize the relative merits of each solution.

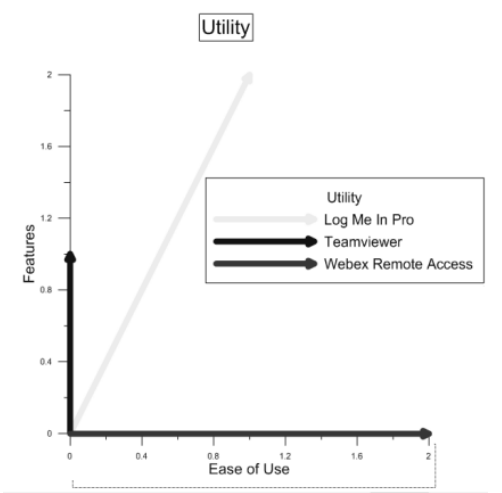




\section{Issues in Information Systems}

Volume 13, Issue 1, pp. 94-104, 2012

\section{Security}

The last major variable to be considered in the selection of a remote access solution is security. Adding remote desktop control software to a computer inherently creates a new threat vector for malicious users to exploit. However, it is not an unacceptable risk for the benefit. The wise stakeholder will want to mitigate the risk when possible. The term security includes many factors such as authentication, encryption, password strength, etc. However, the various factors are not complex enough to warrant creating a sub-tier of variables. Instead the various factors will be considered in aggregate to create a preference ranking. The various unique features and differences will be detailed for each solution. However, all three solutions have some security features in common. All have the capability to audit and log remote sessions. Additionally, all solutions use an SSL or equivalent encryption scheme with public/private keys to ensure end-to-end data security. All use minimum password strength of a 6 character simple password.

Log Me In Pro has the most extensive set of available security features and options of the three solutions. The remote user must authenticate by logging into the Log Me In Pro services before they can initiate a session. This can be bypassed by the host generating an 'invitation', but those invitations are deliberately sent and have a specific timed duration. Secondly, once the session is initiated the remote user is prompted to authenticate to the host operating system. The strength and reliability of this as a security measure is dependent on the security policies of the host system. The two above are the default levels of authentication. However, there are several additional authentication options that can be enabled. A personal password that is stored on the host and never transmitted to the server can be used. In which case, the remote user is asked for only part of the password at the start of each session. For instance, the user may be asked for the $2^{\text {nd }}, 3^{\text {rd }}$, and $5^{\text {th }}$ character from the personal password. Additionally, the user can configure the account to prompt for the entry of an authentication code from a pre-printed list or one generated and emailed at the time the session begins. These codes are randomly generated and each used only one time. These codes are generated, stored and authenticated at the Log Me In Pro server gateway and never transmitted to the host. In addition to these authentication features the software has a configurable lockout feature that temporarily blocks connection attempts after multiple failed logins and a denial of service lockout feature. The client can be configured to be even more restrictive allowing remote access to only certain operating system users or for connections to be allowed from only certain internet source addresses. Lastly, Log Me In Pro can be set to require user permission at the start of each remote session. This makes the computer user the last line of defense in the security environment [11].

TeamViewer has fewer additional security options, but still covers the major bases. By default TeamViewer installs as an attended access program. When functioning in that mode the software uses a new password each time it is launched. These passwords are randomly generated, since they change so quickly are difficult to guess. If the host is configured for unattended access the user specifies a permanent password with a minimum length of 6 characters. The user also needs to know a 9 digit host ID code to attempt to connect to a computer. These ID codes are unique to TeamViewer and are authenticated at each connection attempt. The connecting computer must also have a valid ID code to attempt the connection. TeamViewer has the option to enable Operating System validation for remote users as well. This feature is disabled by default, but does add a second layer of security. If enabled the software can allow or block remote access per user. To avoid brute force attacks for guessing passwords TeamViewer uses exponentially increasing connection latency. After each failed attempt to connect the following attempt takes longer to execute. The artificial delay quickly overcomes the speed advantage of an automated guessing routine. TeamViewer can be set up with the weakest authentication of the three solutions, but it's minimum threshold is less important than it's maximum [16].

WebEx Remote Access base security is sufficient, but lacks many additional security features. It requires authentication when the remote user logs into the central site to initiate a session. However, that authentication can be bypassed by issuing invitations to join a session. These invitations are deliberate and time limited so they do not pose a significant increase in threat. The user is additionally asked to authenticate using a host-specific code at the start of the session. This code is stored and authenticated at the WebEx server, not on the host machine. The 


\section{Issues in Information Systems}

Volume 13, Issue 1, pp. 94-104, 2012

software can be configured to require approval from the host user at the start of the remote session. This introduces a human factor into the security environment. Additionally, WebEx Remote Access can be configured to give remote control over only certain applications rather than over the entire desktop environment. Lastly, connections can be limited to a specific internet source address range. With good decisions this can be a secure application. However, the lack of operating system integration, brute force defense, and one-time use passwords make this the weakest of the three solutions [18].

All of the solutions do make significant provision for computer security. Given the combination of features the preference ranking s are: Log Me In Pro (2), TeamViewer (1), WebEx Remote Access (0).

\section{Summation}

We've now concluded examining all of the variables stated as important in our decision making process. We can combine the information to see which solution is best for the specific context that we have been evaluating. By using the results of the distance formula for each sub-tier we can create new rankings from the 3 top-tier variables.

Summary Ranking Table 1-1

\begin{tabular}{|l|l|l|l|l|l|l|}
\hline & \multicolumn{2}{|l|}{ Log Me In Pro } & TeamViewer & \multicolumn{2}{l|}{ WebEx Remote Access } \\
\hline & Distance & Rank & Distance & Rank & Distance & Rank \\
\hline Cost & 3 & 2 & $2.44948 \ldots$ & 1 & 0 & 0 \\
\hline Utility & $2.360679 \ldots$ & 2 & 1 & 0 & 2 & 1 \\
\hline Security & 2 & 2 & 1 & 1 & 0 & 0 \\
\hline
\end{tabular}

We can create a visualization of our data to see where the strengths of each solution lie. By using the rankings as 3D Coordinates : Log Me In Pro <2, 2,2>, TeamViewer <1,0,1>, WebEx Remote Access <0,1,0>

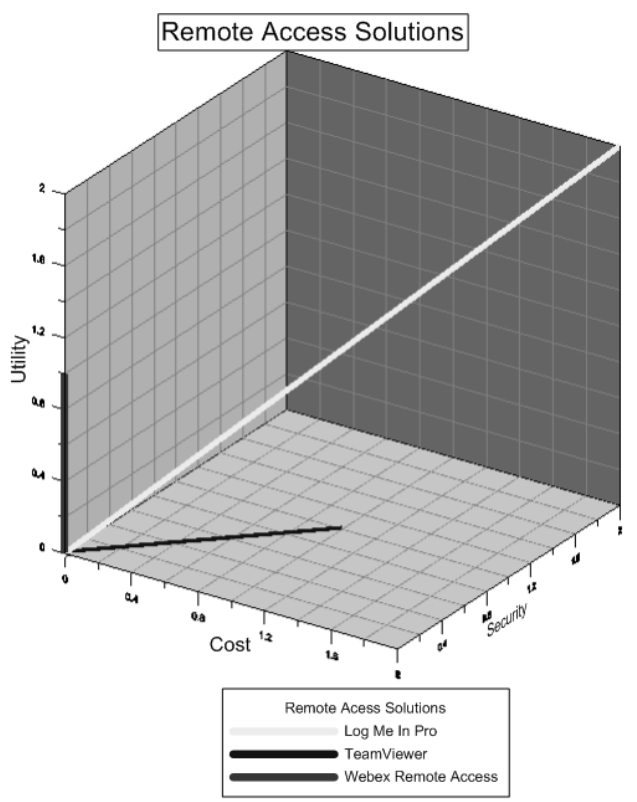

We can apply the distance formula to quantify the relative performance of each solution in this evaluation process. It is important to note that I am not applying any weight factor to any variable. In this instance all variables have an equivalent level of importance in the evaluation. 


\section{Issues in Information Systems}

Volume 13, Issue 1, pp. 94-104, 2012

$\log$ Me In Pro $=\overline{2^{2}+2^{2}+2^{2}}=3.4641016151377545870548926830117$

TeamViewer $=\overline{1^{2}+0^{2}+1^{2}}=1.4142135623730950488016887242097$

WebEx Remote Access $=\overline{0^{2}+1^{2}+0^{2}}=1$

Given these criteria it is obvious that the decision making methodology identifies the Log Me In Pro solution as the best of the presented choices. Evaluating the same software for use in a different environment might produce a different set of variables for consideration, different rankings, or even a weighted rank if some variables within a tier had different levels of importance.

\section{CONCLUSION}

The scope of this paper was twofold. They were to survey remote access solutions for the small business support environment, and to examine a methodology for making complex multivariate business decisions. Log Me In Pro, TeamViewer, and WebEx Remote Access were chosen as 3 potential solutions that fit the basic needs of the environment based on their ability to create an active listening connection that would work in heterogeneous network environments. The methodology for using a recursive distance vector approach for comparing potential solutions was explained. For the specific application of the methodology to this problem the top-tier variables selected were cost, utility, and security. The cost variable was subdivided into 3 scenarios to give a broad idea of how costs would scale over time and with varying number of users and computers. The utility variable combined the concepts of end user ease of use in an attended access scenario, and the features of the solution for the remote support consultant. Security was ranked as an aggregate without sub-dividing the variable further. This example gave us a clear favorite of Log Me In Pro for the specific environment examined. It further demonstrated use of the methodology, showing the use of recursion, aggregate tiers, and combining tiers with a variable number of sub-tiers into a decision model. Given this model a stakeholder can plug in their own preferences and rankings about any number of business decisions and get a concise summarized result, as well as being able to visualize the competing benefits and factors along each step of the process.

\section{REFERENCES}

1. Azad, K. (n.d.) How To Measure Any Distance With The Pythagorean Theorem. In BetterExplained.com. Retrieved April 10, 2012 from http://betterexplained.com/articles/measure-any-distance-with-the-pythagoreantheorem/

2. Bryson, N., \& Ngwenyama, O. (1999). Eliciting and mapping qualitative preferences to numeric rankings in group decision making. European Journal of Operational Research.Vol 116. p. 487-497 Bucatinsky, J. (1996). Technology in small businesses. The CPA Journal, 66(11), 36-38+.

3. Buy LogMeIn Pro. (n.d.) in LogMeIn.com. Retrieved April 10, 2012 from https://secure.logmein.com/products/pro/purchase.aspx

4. Comparison of remote desktop software (n.d.) In Wikipedia. Retrieved April 10, 2012 from http://en.wikipedia.org/wiki/Comparison_of_remote_desktop_software

5. Dassanayaka, T., Dissanayaka, R.,Perer, D., Samarathunga, D., Wijekoon, J., \& Wijesundara, M.(2011). The Advanced Remote PC Management Suit. 6th International Conference on Industrial and Information Systems. Sri Lanka. [Abstract]

6. Gibbs, M. (2009). Managing remote desktop management. Network World, 26(28), 24-24.

7. Humphrey, B. (2008, Making good, effective decisions. Concrete Contractor, 8(3), 86-86.

8. Lifetime License. (n.d.).in TeamViewer.com. Retrieved April 10, 2012 from http://www.teamviewer.com/en/licensing/index.aspx?split=A

9. Log Me In, Inc. (2012). Log Me In Pro User's Guide. Retrieved April 10, 2012 from https://secure.logmein.com/welcome/documentation/EN/pdf/Pro/LogMeIn_Pro_UserGuide.pdf 


\section{Issues in Information Systems}

Volume 13, Issue 1, pp. 94-104, 2012

10. Log Me In, Inc. (2012). Log Me In Security: An In-Depth Look. Retrieved April 10, 2012 from https://secure.logmein.com/welcome/documentation/EN/pdf/common/LogMeIn_SecurityWhitepaper.pdf

11. Miller, I. (September 1975). Graphics in business decision making. Computers \& Graphics. Vol 1. p. 293-296

12. Pash, A. (November 9, 2008). Five Best Remote Desktop Tools. Lifehacker.com. Retrieved from http://lifehacker.com/5080121/five-best-remote-desktop-tools

13. Remote-pc-access.no1reviews.com. (2012) Reviews of the Best Remote PC Access Services. Retrieved from http://remote-pc-access.no1reviews.com/ on 4/10/2012

14. TeamViewer, GmbH. (n.d.) TeamViewer 7 Manual Remote Control. Retrieved April 10, 2012 from http://www.teamviewer.com/en/res/pdf/TeamViewer7_Manual_RemoteControl_EN.pdf

15. TeamViewer, GmbH. (n.d.) TeamViewer Security Statement. Retrieved April 10, 2012 from http://www.teamviewer.com/images/pdf/TeamViewer_SecurityStatement.pdf

16. WebEx Communications, Inc. (2009). Cisco WebEx Support Center Remote Access. Retrieved April 10, 2012 from http://www.wbximg.com/includes/documents/data-sheets/ra_ds.pdf

17. WebEx Communications, Inc. (n.d.). WebEx Support CenterRemote Access Security. Retrieved April 10, 2012 from http://www.webex.com/pdf/wp sc remote access security.pdf

18. Wildstrom, S. H. (2005). Beaming Into Your PC From Anywhere. Businessweek, (3923), 26. 